\title{
A New Extended Padé Approximation and Its Application
}

\author{
Z. Kalateh Bojdi, ${ }^{1}$ S. Ahmadi-Asl, ${ }^{1}$ and A. Aminataei ${ }^{2}$ \\ ${ }^{1}$ Department of Mathematics, Birjand University, Birjand, Iran \\ ${ }^{2}$ Faculty of Mathematics, K. N. Toosi University of Technology, P.O. Box 16315-1618, Tehran, Iran
}

Correspondence should be addressed to A. Aminataei; ataei@kntu.ac.ir

Received 19 June 2013; Revised 16 September 2013; Accepted 7 October 2013

Academic Editor: Weimin Han

Copyright (C) 2013 Z. Kalateh Bojdi et al. This is an open access article distributed under the Creative Commons Attribution License, which permits unrestricted use, distribution, and reproduction in any medium, provided the original work is properly cited.

\begin{abstract}
We extend ordinary Padé approximation, which is based on a set of standard polynomials as $\left\{1, x, \ldots, x^{n}\right\}$, to a new extended Padé approximation (Müntz Padé approximation), based on the general basic function set $\left\{1, x^{\lambda}, x^{2 \lambda}, \ldots, x^{n \lambda}\right\}(0<\lambda \leq 1)$ (the particular case of Müntz polynomials) using general Taylor series (based on fractional calculus) with error convergency. The importance of this extension is that the ordinary Padé approximation is a particular case of our extended Padé approximation. Also the parameterization ( $\lambda$ is the corresponding parameter) of new extended Padé approximation is an important subject which, obtaining the optimal value of this parameter, can be a good question for a new research.
\end{abstract}

\section{Introduction}

Rational approximations of an arbitrary function are an important topic in numerical analysis due to their high applications in physical sciences, chemistry, engineering, and other applied sciences $[1,2]$. The Padé approximation is a particular and classical type of rational fraction approximation. The idea of this approximation is to expand a function as a ratio of two power series and determining both the numerator and denominator coefficients using the coefficients of Taylor series expansion of a function $f(x)$ [1]. The Padé approximation is the best approximation of a function by a rational function of a given order [1]. The technique was developed around 1890 by Henri Padé, but it goes back to George Freobenius who introduced the idea and investigated the features of rational approximations of power series. The Padé approximation is usually superior when functions contain poles, because the use of rational function allows them to be well represented [1]. The Padé approximation often gives better approximation of the function than truncating its Taylor series, and it may still work where the Taylor series does not converge [1]. For these reasons, Padé approximation is used extensively in computer calculations. The Padé approximation has also been used as an auxiliary function in Diophantine approximation and transcendental number theory, though for sharp results ad hoc methods in some sense inspired by the Padé theory typically replace them. Since it provides an approximation to the function throughout the whole complex plane, the study of Pade approximation is simultaneously a topic in mathematical approximation theory and analytic function theory. The generalized Padé approximation is given in [2]. For the connection of Padé approximation with continued fractions and orthogonal polynomials, see [2]. Also multivariate Padé approximation was done by [3]. Two versions of this approximation which concerned with interpolation problems are Hermite-Padé and Newton-Padé approximations and they are in fact rational interpolation problems [4-6]. In this paper, using the generalized Taylor series (based on fractional calculus), we extend ordinary Padé approximation to the general Müntz padé approximation. The paper is organized as follows. In Sections 1.1 to 1.3 , we introduce an ordinary Padé approximation (and their properties), present the Müntz polynomials (rational Müntz functions and suitable theorems), and also generalize Taylor series (based on fractional calculus), respectively. Also Müntz Padé approximation is presented in Section 2. The uniqueness and convergence analysis results are given in Section 3. Some numerical tests are presented in Section 4. Two applications of this extended Padé approximation (Müntz Padé approximation) on the numerical solution of sound and vibration problem and microwave heating model in a slab using differential equations are given in 
Section 5. Finally we have monitored a brief conclusion in Section 6.

1.1. Ordinary Padé Approximation. Suppose that we are given a power series $\sum_{i=0}^{\infty} c_{i} z^{i}$, represent a function $f(z)$, so that

$$
f(z)=\sum_{i=0}^{\infty} c_{i} z^{i}
$$

This expansion is the fundamental starting point of any analysis using Padé approximation. A Padé approximation is a rational fraction as

$$
\left[\frac{L}{M}\right]=\frac{a_{0}+a_{1} z+\cdots+a_{L} z^{L}}{b_{0}+b_{1} z+\cdots+b_{M} z^{M}}
$$

which has a Maclaurin expansion which agrees with (1) as far as possible. Notice that, in (2), there are $L+1$ numerator coefficients and $M+1$ denominator coefficients. There is a more or less irrelevance common factor between them, and for definiteness we take $b_{0}=1$. So there are $L+1$ independent numerator coefficients and $M$ independent denominator coefficients, making $M+L+1$ unknown coefficients in all. This number suggests that normally the $[L / M]$ out to fit the power series (1) through the orders $1, z, \ldots, z^{L+M}$. For the notation of formal power series, we have

$$
\sum_{i=0}^{\infty} c_{i} z^{i}=\frac{a_{0}+a_{1} z+\cdots+a_{L} z^{L}}{b_{0}+b_{1} z+\cdots+b_{M} z^{M}}+O\left(z^{L+M+1}\right),
$$

and returning to (3) and cross-multiplying, we find that

$$
\begin{aligned}
& \left(b_{0}+b_{1} z+\cdots+b_{M} z^{M}\right)\left(c_{0}+c_{1} z+\cdots\right) \\
& =a_{0}+a_{1} z+\cdots+a_{L} z^{L}+O\left(z^{L+M+1}\right) .
\end{aligned}
$$

Equating the coefficients of $z^{L+1}, z^{L+2}, \ldots, z^{L+M}$ from (4), we find

$$
\begin{gathered}
b_{M} c_{L-M+1}+b_{M-1} c_{L-M+2}+\cdots+b_{0} c_{L+1}=0 \\
b_{M} c_{L-M+2}+b_{M-1} c_{L-M+3}+\cdots+b_{0} c_{L+1}=0 \\
\vdots \quad \vdots \\
b_{M} c_{L}+b_{M-1} c_{L-M+1}+\cdots+b_{0} c_{L+M}=0 .
\end{gathered}
$$

If $j<0$, we define $c_{j}=0$ for consistency. Since $b_{0}=1$, (5) becomes a set of $M$ linear equations for the $M$ unknown denominator coefficients and we obtain

$$
\left[\begin{array}{cccc}
c_{L-M+1} & c_{L-M} & \ldots & c_{L} \\
c_{L-M+2} & c_{L-M+1} & \ldots & c_{L+1} \\
\vdots & \vdots & \ddots & \vdots \\
c_{L} & c_{L+1} & \ldots & c_{L+M-1}
\end{array}\right]\left[\begin{array}{c}
b_{M} \\
b_{M-1} \\
\vdots \\
b_{1}
\end{array}\right]=-\left[\begin{array}{c}
c_{L+1} \\
c_{L+2} \\
\vdots \\
c_{L+M}
\end{array}\right]
$$

from which the $b_{i}$ may be found. The numerator coefficients $a_{0}, \ldots, a_{L}$ follow immediately from (4) by equating the coefficients of $1, z, \ldots, z^{L}$ as

$$
\begin{gathered}
a_{0}=c_{0}, \\
a_{1}=c_{1}+c_{0}, \\
a_{2}=c_{2}+b_{1} c_{1}+b_{2} c_{0}, \\
\vdots \\
a_{l}=c_{l}+\sum_{i=0}^{\min \{L, M\}} b_{i} c_{L-i} .
\end{gathered}
$$

Thus (4) and (7) normally determine the Padé numerator and denominator which are called Padé equations and we have constructed an $[L / M]$ Padé approximation which agrees with $\sum_{i=0}^{\infty} c_{i} z^{i}$ through order $z^{L+M}$. If the given power series converge to the same function for $|z|<R$ with $(0<R<+\infty)$, then a sequence of Padé approximation may converge for $z \in D$, where $D$ is a domain longer than $|z|<R[1]$.

Remark 1. In general, Padé approximation does not exist (for an arbitrary function with respect to particular $L$ and $M$ ) [1].

Theorem 2. If the Padé approximation $[L / M]$ exists, then it will be unique [2].

1.2. Müntz Polynomials. An interesting generalization of Weierstrass theorem (1885) (density of polynomials set in $C[a, b]$, equipped with a supremum norm) goes back to the German mathematician Herman Müntz. He performed this generalization by a suitable tool which is named Müntz polynomials. For presenting his theorem, first we introduce a definition and a remark.

Definition 3. Suppose $\lambda_{0}<\lambda_{1}<\cdots \in \mathbb{R}$; then, the set $\left\{x^{\lambda_{0}}, x^{\lambda_{1}}, \ldots\right\}$ is named as a set of Müntz or Müntz polynomials [6].

Remark 4. Let $\left(\lambda_{i}\right)_{i=0}^{\infty}$ be any sequences of distinct real numbers and $a>0$; then,

$$
\left\{\frac{\sum_{i=0}^{n} a_{i} x^{\lambda i}}{\sum_{i=0}^{n} b_{i} x^{\lambda i}} ; a_{i}, b_{i} \in \mathbb{R}, n \in \mathbb{N}\right\},
$$

is dense in $C[a, b][6]$.

Theorem 5 (generalized Müntz theorem). If $0 \leq \lambda_{0}<\lambda_{1}<$ $\cdots \rightarrow+\infty$, then the Müntz polynomials with respect to these sequences are dense in $L^{2}[0,1]$, if and only if $\sum_{i=0}^{\infty} \lambda_{i}^{-1}=\infty$ [6].

Remark 6. In this paper, by considering $\lambda_{k}=k \lambda, k=$ $1,2, \ldots(0<\lambda \leq 1)$ which is a particular case of Müntz polynomials, we introduce a new and extended Müntz Padé approximation. Also, it is clear that by considering $\lambda_{k}=k$, we can obtain the classical Weierstrass theorem [6]. 
1.3. Generalized Taylor Series. In calculus, Taylor's theorem gives an approximation of a $k$-times differentiable function $f(x)$ around a given point $x_{0}$ by a $k$ th order Taylor polynomial as

$$
f(x) \simeq \sum_{k=0}^{n} \frac{f^{(k)}\left(x_{0}\right)}{k !}\left(x-x_{0}\right)^{k}
$$

with the error

$$
e(x)=\frac{f^{(n+1)(\varsigma)}}{(n+1) !}\left(x-x_{0}\right)^{n+1}, \quad \varsigma \in\left(x_{0}, x\right),
$$

and for analytical polynomials, we have

$$
f(x)=\sum_{k=0}^{\infty} \frac{f^{(k)}\left(x_{0}\right)}{k !}\left(x-x_{0}\right)^{k} .
$$

Taylor's theorem also generalizes to multivariate and vector valued functions. But the generalized version of this theorem from fractional calculus view or the theory of derivatives of arbitrary order was done by [7], because of its high applications in solving fractional ordinary differential equations integral ordinary differential equations and obtaining an operational matrix, and so forth. For this purpose, we need some definitions in fractional calculus.

Definition 7. The Caputo fractional derivative of $f(x)$ of order $\lambda>0$ with $a \geq 0$ is defined as

$$
\left(D_{a}^{\lambda} f\right)(x)=\frac{1}{\Gamma(\beta-\lambda)} \int_{a}^{x} \frac{f^{(\beta)}(t)}{(x-t)^{\lambda-\beta+1}} d t,
$$

for $\beta-1<\lambda \leq \beta, \beta \in \mathbb{N}, x \geq a$.

From Caputo fractional derivative, we have

$$
\begin{gathered}
D_{a}^{\lambda} C=0, \\
D_{a}^{\lambda} x^{\delta}= \begin{cases}0, & \text { if } \delta \in\{1, \ldots, \beta-1\}, \\
\frac{\Gamma(\delta+1)}{\Gamma(\delta+1-\lambda)}(x)^{\delta-\lambda}, & \text { if } \delta \in \mathbb{N}, \delta \geq \beta, \\
& \text { or } \delta>\beta-1,\end{cases}
\end{gathered}
$$

where $\beta=\lceil\lambda\rceil$ and $\lambda \geq 0$. However, the Caputo fractional derivative is a linear operator which means

$$
D_{a}^{\lambda}(\gamma f+\theta g)=\gamma D_{a}^{\lambda}(f)+\theta D_{a}^{\lambda}(g) .
$$

For more literature review of fractional calculus, see [8].

Theorem 8. Suppose that $D_{a}^{k \lambda} f(x) \in C(a, b]$, for $k=$ $0,1, \ldots, n+1$, where $0<\lambda \leq 1$; then, one has

$$
\begin{aligned}
f(x)= & \sum_{k=0}^{n} \frac{D_{a}^{k \lambda} f(a)}{\Gamma(\lambda k+1)}(x-a)^{k \lambda} \\
& +\frac{D_{a}^{(n+1) \lambda} f(\varsigma)}{\Gamma((n+1) \lambda+1)}(x-a)^{(n+1) \lambda},
\end{aligned}
$$

where $a \leq \varsigma \leq b$, for all $x \in(a, b]$, and $D_{a}^{(n) \lambda}=D_{a}^{\lambda} \cdot D_{a}^{\lambda} \cdots D_{a}^{\lambda}$, (n-times) [7].
If we consider $a=0$, then we obtain a fractional Maclaurin series and in a similar manner as the arbitrary function $f(z)$ that has an infinite Caputo differentiable at point $a$ named as analytical functions on $D$ in a fractional sense if

$$
f(z)=\sum_{k=0}^{\infty} \frac{D_{a}^{k \lambda} f(a)}{\Gamma(\lambda k+1)}(z-a)^{k \lambda}, \quad \forall z \in D .
$$

\section{Müntz Padé Approximations}

Now by using the general Taylor series (based on fractional calculus), we generalize the classical and ordinary Padé approximations to a new and extended Müntz Padé approximation. For this goal, we present the Müntz Padé approximation as a ratio of two Müntz polynomials constructed from the coefficients of generalized Taylor series expansion of a function. Also in a similar approach from ordinary Padé approximation, we prove the uniqueness of Müntz Padé approximation. Now in a similar manner from ordinary Padé approximation, suppose that $f(x)$ is an analytical function (from fractional calculus) in the neighborhood of $a=0$, and then we can write $f(x)$

$$
f(x)=\sum_{k=0}^{\infty} c_{k} z^{\lambda k}, \quad c_{k}=\frac{D_{a}^{k \lambda}}{\Gamma(k \lambda+1)},
$$

and also Müntz Padé approximation is defined as a ratio of two Müntz polynomials as

$$
\left[\frac{L}{M}\right]=\frac{a_{0}+a_{1} z^{\lambda}+\cdots+a_{L} z^{L \lambda}}{b_{0}+b_{1} z^{\lambda}+\cdots+b_{M} z^{M \lambda}},
$$

and then we suppose that $[L / M]$ (Müntz Padé approximation) has a Maclaurin series (17) or

$$
\sum_{i=0}^{\infty} c_{i} z^{\lambda i}=\frac{a_{0}+a_{1} z^{\lambda}+\cdots+a_{L} z^{L \lambda}}{b_{0}+b_{1} z^{\lambda}+\cdots+b_{M} z^{M \lambda}}+O\left(z^{L \lambda+M \lambda+1}\right) .
$$

By cross multiplying, we find that

$$
\begin{aligned}
\left(b_{0}+\right. & \left.b_{1} z^{\lambda}+\cdots+b_{M} z^{M \lambda}\right)\left(c_{0}+c_{1} z^{\lambda}+\cdots\right) \\
& =a_{0}+a_{1} z^{\lambda}+\cdots+a_{L} z^{L \lambda}+O\left(z^{L \lambda+M \lambda+1}\right) .
\end{aligned}
$$

Equating the coefficients $z^{\lambda L+\lambda}, z^{\lambda L+2 \lambda}, \ldots, z^{\lambda L+\lambda M}$, from (20) we obtain a system of equations similar to (5) and we can obtain $b_{i}$ coefficients. Also immediately by equating the coefficients of $1, z^{\lambda}, \ldots, z^{L \lambda}$, we can obtain $a_{i}$ coefficients in a recursion formula as (7). Thus by assumption of existence of $[L / M]$ Müntz Padé approximation, for establishing the uniqueness of this approximation, we must prove that the matrix of system of (5) is nonsingular, but we perform the proof of the uniqueness of Müntz Padé approximation by a different approach in the next section.

Remark 9. Let $\lambda=1$; then, we can obtain the ordinary Padé approximation. 


\section{Uniqueness and Convergence Analysis of Müntz Padé Approximation}

In this section, we present the uniqueness and convergence analysis of Müntz Padé approximation in some theorems.

Theorem 10 (uniqueness). When Müntz Padé approximation $([L / M])$ exists, then it is unique for any formal power series.

Proof. Assume that there are two such Müntz Padé approximations $U(z) / V(z)$ and $X(z) / Y(z)$, where the degrees of $X$ and $U$ are less than or equal to $\lambda L$ and that of $Y$ and $V$ are less than or equal to $\lambda M$. Then by (19), we must have

$$
\frac{X(z)}{Y(z)}-\frac{U(z)}{V(z)}=O\left(z^{L \lambda+M \lambda+1}\right)
$$

since both approximate the same series. If we multiply (21) by $Y(z) V(z)$, we obtain

$$
X(z) V(z)-Y(z) U(z)=O\left(z^{L \lambda+M \lambda+1}\right),
$$

but the left-hand side of (22) is a polynomial of degree at most $\lambda L+\lambda M$ and thus is identically zero. Since neither $Y$ nor $V$ is identically zero, we conclude

$$
\frac{X(z)}{Y(z)}=\frac{U(z)}{V(z)}
$$

Since, by definition, both $X$ and $Y$ and $U$ and $V$ are relatively prime and $Y(0)=V(0)=1$; we have shown that the two supposedly different Müntz Padé approximants are the same.

Theorem 11 (convergency). Let $f(z)$ be analytic in $|z| \leq R$ (in fractional sense); then, an infinite subsequence of [L/1] Müntz Padé approximants converges to $f(z)$ uniformly in $|z| \leq R$.

Proof. By hypothesis, $f(z)$ is analytic in $|z| \leq R$ (in fractional sense) and consequently within a large interval, $|z|<\rho$, with $\rho>\rho^{\prime}>R$. Let

$$
f(z)=\sum_{i=0}^{\infty} c_{i} z^{\lambda i}, \quad \text { with } c_{i}=O\left(\left(\rho^{\prime}\right)^{-\lambda i}\right) .
$$

The $[L / 1]$ Müntz Padé approximation is given by

$$
\left[\frac{L}{1}\right]=c_{0}+c_{1} z^{\lambda}+\cdots+c_{L-1} z^{\lambda(L-1)}+\frac{c_{L} z^{\lambda L}}{1-c_{L+1} z^{\lambda} / c_{L}} .
$$

If a subsequence of coefficients $\left\{c_{L_{j}}, j=1,2, \ldots\right\}$ are zero, then $\left[\left(L_{j}-1\right) / 1\right]$ are truncated fractional Maclaurin expansions which converge to $f(z)$ uniformly in $|z| \leq R<\rho^{\prime}$. So we assume that no infinite subsequence of $\left\{c_{L}\right\}$ vanishes and consider $r_{L}=c_{L+1} / c_{L}$ which is well defined for all sufficiently large $L$, because

$$
c_{L} z^{\lambda L}=O\left(\frac{R}{\rho^{\prime}}\right)^{\lambda L}
$$

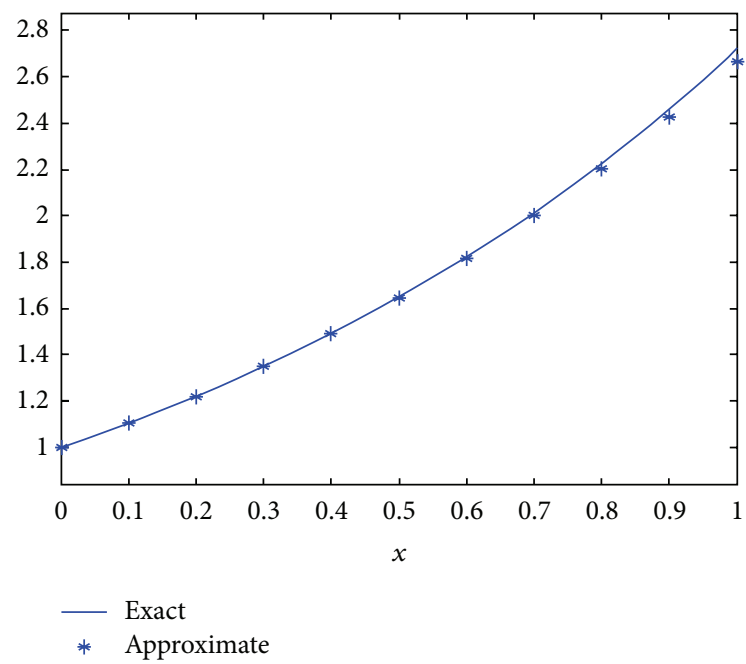

Figure 1: The [3/5] Müntz Padé approximation of $f(x)=e^{x}$ and exact function of $\lambda=0.4$.

the sequence of $[L / 1]$ Müntz Padé approximation given by (25) converges uniformly in $|z|<R$ unless there exists a sequence of values of $L$ for which $1-c_{L+1} z / c_{L}=0$ within $|z|<$ $\rho^{\prime}$. Thus either a subsequence of the second row converges uniformly or else for some $L_{0}$ and all $L>L_{0},\left|c_{L} / c_{L+1}\right|>\rho^{\prime}$. In the latter case

$$
\left|\frac{c_{L_{0}}}{c_{L}}\right|=\prod_{i=L_{0}}^{L-1}\left|\frac{c_{i}}{c_{i+1}}\right|>\left(\rho^{\prime}\right)^{\lambda\left(L-L_{0}\right)},
$$

contradicting with (24), so the proof is completed.

\section{The Test Experiments}

Now in this section, in the two subsections, we show the applications of the Müntz Padé approximation in the functional approximation (see Section 4.1) and fractional calculus fields. The advantage of using the Müntz Padé approximation is shown for numerical approximation of fractional differential equations in Section 4.2.

4.1. Müntz Padé Approximation and Functional Approximation. In this section, we obtain the Müntz Padé approximations of $f(x)=e^{x}$, for different values of $\lambda$. Figure 1 shows the [3/5] Müntz Padé approximations of $f(x)=e^{x}$ and $\lambda=0.4$.

Also the [3/5] Müntz Padé approximation of $f(x)=e^{x}$, and $\lambda=0.65$ is shown in Figure 2 .

4.2. Müntz Padé Approximation and Fractional Calculus. This section is devoted to presentation of some numerical simulations obtained by applying the collocation method and based on a new extended Padé approximation (Müntz Padé approximation). The algorithm for numerical approximation of solutions to the initial value problems for the fractional differential equations was implemented by MATLAB. In the case of nonlinear equations, the MATLAB function $f$ solve was 


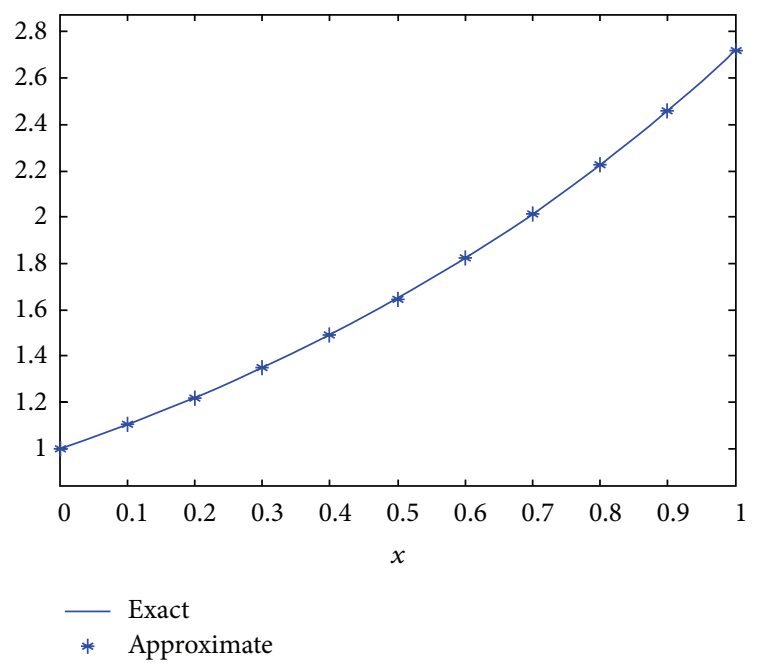

Figure 2: Comparison between the [3/5] Müntz Padé approximation of $f(x)=e^{x}$ and exact function of $\lambda=0.65$.

used for solving the nonlinear system. In the case that the exact solution $y$ to a problem is known, the dependence of approximation errors on the discretization parameter $n$ is estimated in 2-norm as

$$
e_{n}=\sqrt{\sum_{k=0}^{n}\left(y_{n}\left(x_{k}\right)-y\left(x_{k}\right)\right)^{2}},
$$

where $y_{n}$ is the approximated solution corresponding to the discretization parameter $n$.

Experiment 1. We start with a simple nonlinear problem [8]

$$
\begin{aligned}
D^{\alpha}(y(x))= & \frac{40320}{\Gamma(9-\alpha)} x^{8-\alpha}-3 \frac{\Gamma(5+\alpha / 2)}{\Gamma(5-\alpha / 2)} x^{4-\alpha / 2} \\
& +\frac{9}{4} \Gamma(\alpha+1)+\left(\frac{3}{2} x^{\alpha / 2}-x^{4}\right)^{3}-(y(x))^{3 / 2},
\end{aligned}
$$

where we have a nonlinear and nonsmooth right-hand side. The solution $y$ has a smooth derivative of order $0<\alpha<1$ [8]. The analytical solution subject to the initial condition $y(0)=$ 0 is given by

$$
y(t)=x^{8}-3 x^{4+\alpha / 2}+\frac{9}{4} x^{\alpha} .
$$

Now we approximate the exact solution of (29) or $y^{\lambda}(x)$, with the $[n / 1]$ new and extended Padé approximation (Müntz Padé approximation) as

$$
y^{\lambda}(x) \simeq y_{n}^{\lambda}(x)=\sum_{i=0}^{n} c_{i} x^{i \lambda}
$$

and substituting it into (29), we obtain

$$
\operatorname{Res}_{n}^{(\alpha, \lambda)}(x)=D^{\alpha}\left(y_{n}^{\lambda}(x)\right)-P(\alpha, y(x), x) \simeq 0,
$$

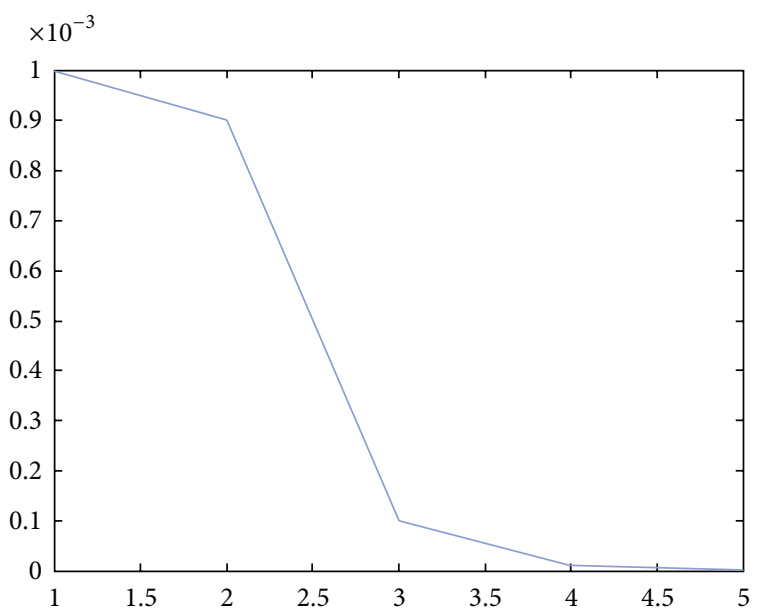

Figure 3: [12/1] ordinary Padé approximation coefficients of $\alpha=$ $0.5, \beta=0.25$, and $\gamma=0.75$ of Experiment 1 .

where

$$
\begin{aligned}
P(\alpha, y(x), x)= & \frac{40320}{\Gamma(9-\alpha)} x^{8-\alpha}-3 \frac{\Gamma(5+\alpha / 2)}{\Gamma(5-\alpha / 2)} x^{4-\alpha / 2} \\
& +\frac{9}{4} \Gamma(\alpha+1)+\left(\frac{3}{2} x^{\alpha / 2}-x^{4}\right)^{3}-(y(x))^{3 / 2} .
\end{aligned}
$$

For the collocation points, we use the first roots of the Jacobi polynomial $P_{n}^{(\beta, \gamma)}(x)[9,10]$, and then after enforcing the initial condition $y(0)=0$, we obtain a system of nonlinear algebraic equations and we use the MATLAB function $f$ solve for solving the nonlinear system. Thus, substituting the collocation points into (32) yields

$$
\begin{aligned}
\operatorname{Res}_{n}^{(\alpha, \lambda)}\left(x_{i}^{(\beta, \gamma)}\right)= & D^{\alpha}\left(y_{n}^{\lambda}\left(x_{i}^{(\beta, \gamma)}\right)\right) \\
& -P\left(\alpha, y\left(x_{i}^{(\beta, \gamma)}\right), x_{i}^{(\beta, \gamma)}\right) \simeq 0, \\
& i=0, \ldots, n .
\end{aligned}
$$

Now from (34) and its initial condition, we have $n+2$ algebraic equations of $n+1$ unknown coefficients. Thus for obtaining the unknown coefficients, we must eliminate one arbitrary equation from these $n+2$ equations. But because of the necessity of holding the boundary conditions, we eliminate the last equation from (34). Finally, replacing the last equation of (34) by the equation of initial condition, we obtain a system of $n+1$ equations of $n+1$ unknowns $c_{i}$. By implementing the method as presented, for $n=5$ and also for different parameters of $\beta$ and $\gamma$, we obtain the approximate solutions. The $[12 / 1]$ ordinary and Müntz Padé coefficients approximation of this problem are shown in Figures 3 and 4, respectively. We have observed that this method (the new extended Padé approximation (Müntz Padé approximation)) is very efficient for numerical approximation of the fractional ordinary differential equations. Also, closer look at the results of the Müntz Padé approximation scheme reveals that in this method of solution the coefficients decrease faster than the classical case. 


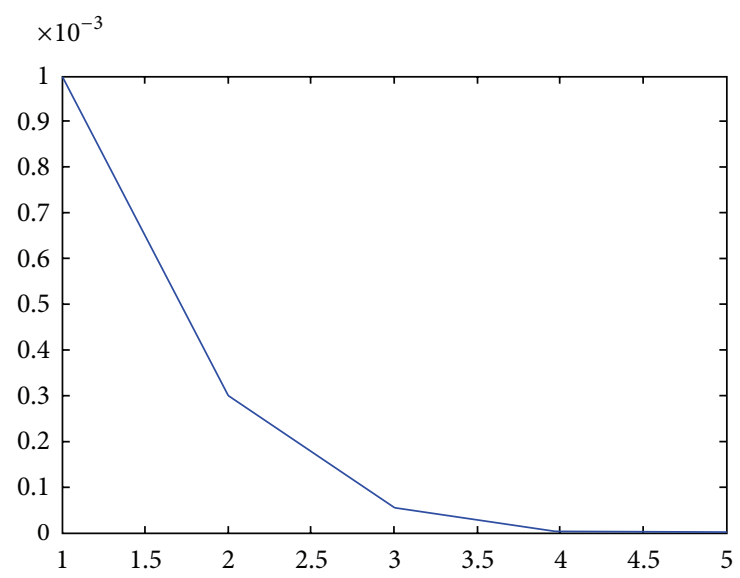

Figure 4: [12/1] new Padé approximation coefficients of $\lambda=0.5$, $\alpha=0.5, \beta=0.25$, and $\gamma=0.75$ of Experiment 1 .

This is the advantage on the application of the Müntz Padé approximation to the fractional ordinary differential equations.

Experiment 2. Consider the nonlinear fractional integrodifferential equation [11]

$$
\begin{array}{r}
D^{1 / 2} u(x)=f(x) u(x)+g(x)+\sqrt{x} \int_{0}^{x} u^{2}(t) d t \\
y(0)=0
\end{array}
$$

where

$$
\begin{gathered}
f(x)=2 \sqrt{x}+2 x^{3 / 2}-\left(\sqrt{x}+x^{3 / 2}\right) \ln (1+x), \\
g(x)=\frac{2 \arcsin h(\sqrt{x})}{\sqrt{\pi} \sqrt{1+x}}-2 x^{3 / 2} .
\end{gathered}
$$

The exact solution of $(35)$ is $u(x)=\ln (1+x)$. We implement the collocation method based on the [10/1] new Padé approximation of $\lambda=0.16, \beta=1$, and $\gamma=1$. The obtained results of our method are presented in Table 1 .

\section{Application of Müntz Padé Approximation in Vibration and Electromagnetic Radiation Problems}

In this section, we present two applications of Müntz Padé approximation for numerical approximation of some applicable ordinary differential equations.

Experiment 3. Consider the following model problem [1215]:

$$
\frac{d x}{d t}=\alpha x+\varepsilon x^{2}, \quad x(0)=1,
$$

TABLE 1: The comparison between the collocation method based on [10/1] Müntz Padé approximation and the exact solution of $\lambda=$ $0.16, \beta=1, \gamma=1$, of Experiment 2.

\begin{tabular}{lccc}
\hline$x$ & Exact & Approximate & Error \\
\hline 0.0 & 0.0000000000 & 0.0000000000 & 0.000000000 \\
0.1 & 0.0953101798 & 0.0953103008 & 0.000000121 \\
0.2 & 0.18232155679 & 0.1823218407 & 0.000000284 \\
0.3 & 0.26236426446 & 0.2623649824 & 0.000000718 \\
0.4 & 0.33647223662 & 0.3364726886 & 0.000000452 \\
0.5 & 0.40546510810 & 0.40546528510 & 0.000000177 \\
0.6 & 0.47000362924 & 0.4700043522 & 0.000000723 \\
0.7 & 0.53062825106 & 0.5306290280 & 0.000000777 \\
0.8 & 0.58778666490 & 0.58778755290 & 0.000000888 \\
0.9 & 0.64185388617 & 0.64185410017 & 0.000000214 \\
1.0 & 0.69314718055 & 0.69314789955 & 0.000000719 \\
\hline
\end{tabular}

TAble 2: Comparison between the [2/3] and [4/4] Müntz Padé approximations of $\alpha=0.5, \varepsilon=0.4$, and $\lambda=0.5$ of Experiment 3 .

\begin{tabular}{lcc}
\hline$t$ & Error of $[2 / 3]$ & Error of [4/4] \\
\hline 0.0 & 0.0012 & 0.0009 \\
0.1 & 0.0010 & -0.0004 \\
0.2 & 0.0013 & 0.0006 \\
0.3 & 0.0022 & -0.0002 \\
0.4 & 0.0014 & 0.0007 \\
0.5 & 0.0012 & 0.0003 \\
\hline
\end{tabular}

where $0<\varepsilon \leq \alpha \leq 1$ [12]. This model has high application in the theory of sound and vibration [12]. The exact solution to this initial value problem has the form

$$
x(t)=\frac{\alpha \exp (\alpha t)}{(\alpha+\varepsilon-\varepsilon \exp (\alpha t))} .
$$

Now for $\alpha=0.5, \varepsilon=0.4$, and $\lambda=0.5$ and using [2/3] and [4/4] Müntz Padé approximations, we obtain the following results shown in Table 2 .

Also the obtained results for different kinds of Müntz Padé approximations are shown in Figure 5.

Experiment 4. Consider the following model problem [16]:

$$
\frac{d^{2} T}{d y^{2}}+\lambda e^{-k y} T=0
$$

with

$$
\frac{d T}{d y}(0)=0, \quad T(1)=1 .
$$

This equation has high application in the theory of electromagnetic radiation and describes the steady state reactiondiffusion equations with source term that arise in modeling microwave heating in an infinite slab with isothermal walls [16]. Also $\lambda$ and $k$ represent the thermal absorptivity and 


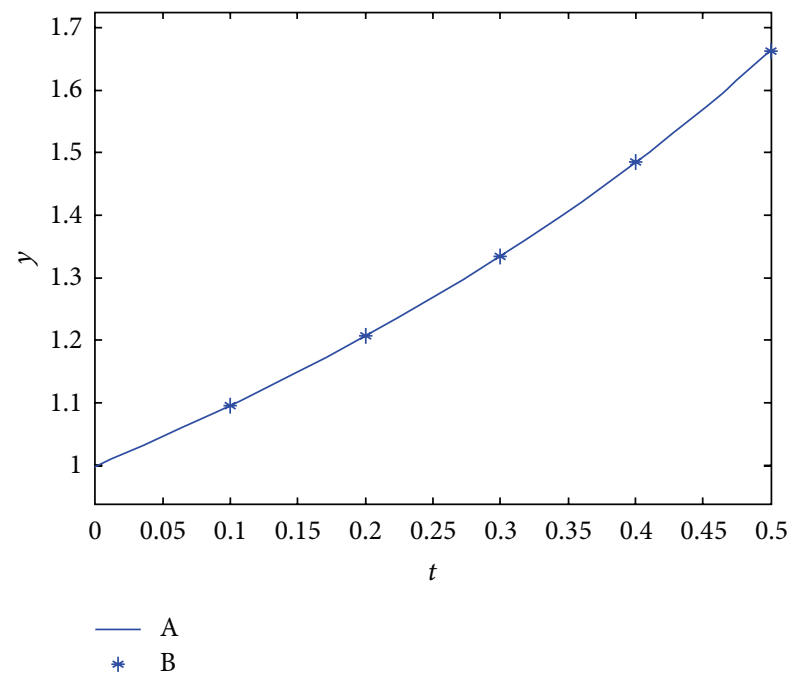

Figure 5: A: the obtained results of [4/4] Müntz Padé approximation of experiment 3. B: the obtained results of [2/3] Müntz Padé approximation of Experiment 3.

Table 3: Comparison between the [2/3] and [4/4] Müntz Padé approximations of $k=1$ and $\lambda=0.5$ of Experiment 4 .

\begin{tabular}{lcc}
\hline$y$ & Error of $[2 / 3]$ & Error of $[4 / 4]$ \\
\hline 0.0 & 0.0002 & 0.0001 \\
0.1 & -0.0033 & -0.0011 \\
0.2 & 0.0014 & 0.0009 \\
0.3 & 0.0003 & 0.0001 \\
0.4 & 0.0026 & -0.0012 \\
0.5 & 0.0023 & 0.0021 \\
\hline
\end{tabular}

electric field decay rate parameters, respectively. The exact solution to this boundary value problem has the form

$$
\begin{aligned}
T(y)= & \left(J_{1}(2(\sqrt{\lambda} / k)) Y_{0}\left(-2\left(\sqrt{\lambda} / k \sqrt{e^{k y}}\right)\right)\right) \\
\times & \left(J_{1}(2(\sqrt{\lambda} / k)) Y_{0}\left(-2\left(e^{-k / 2} \sqrt{\lambda} / k\right)\right)\right. \\
& \left.+Y_{1}(-2(\sqrt{\lambda} / k)) J_{0}\left(2\left(e^{-k / 2} \sqrt{\lambda} / k\right)\right)\right)^{-1} \\
+ & \left(Y_{1}(2(\sqrt{\lambda} / k)) J_{0}\left(-2\left(\sqrt{\lambda} / k \sqrt{e^{k y}}\right)\right)\right) \\
\times & \left(J_{1}(2(\sqrt{\lambda} / k)) Y_{0}\left(-2\left(e^{-k / 2} \sqrt{\lambda} / k\right)\right)\right. \\
& \left.+Y_{1}(-2(\sqrt{\lambda} / k)) J_{0}\left(2\left(e^{-k / 2} \sqrt{\lambda} / k\right)\right)\right)^{-1},
\end{aligned}
$$

where $J_{0}, J_{1}$ are Bessel functions of the first kind and $Y_{0}, Y_{1}$ are Bessel functions of the second kind. Now for $k=1$, and $\lambda=$ 0.5 and using [2/3] and [4/4] Müntz Padé approximations, we obtain the following results shown in Table 3.

\section{Conclusion}

In this paper, using the general Taylor series (based on fractional calculus), we extend the ordinary Padé approximation to the general Müntz Padé approximation. The importance of this extension is that the ordinary Padé approximation is a particular case of our Müntz Padé approximation $(\lambda=1)$. We have applied the method in the application of functional approximation, fractional exponent, and vibration and electromagnetic radiation model problems and have obtained the results with a good order of accuracy. Also the uniqueness results and error analysis have been presented completely. In addition, the test experiments have been presented for showing the applicability and validity of the new Müntz Padé approximation.

\section{Acknowledgments}

The authors are grateful to the reviewers and the editor Professor Dr. Weimin Han for their helpful comments and suggestions which indeed improved the quality of this paper.

\section{References}

[1] G. A. Baker and P. Graves-Morris, Padé Approximants, Addison-Wesley, 1981.

[2] G. A. Baker, Essentials of Padé Approximants, Academic Press, New York, NY, USA, 1975.

[3] A. Cuyt, "Multivariate Padé approximants revisited," BIT, vol. 26, no. 1, pp. 71-79, 1986.

[4] L. Wuytack, "On the osculatory rational interpolation problem," Mathematics and Computers in Simulation, vol. 29, pp. 837-843, 1975.

[5] A. A. M. Cuyt and B. M. Verdonk, "General order NewtonPadé approximants for multivariate functions," Numerische Mathematik, vol. 43, no. 2, pp. 293-307, 1984.

[6] P. Borwein and T. Erdélyi, Polynomials and Polynomials Inequalities, vol. 161 of Graduate Texts in Mathematics, Springer, Berlin, Germany, 1995.

[7] Z. M. Odibat and N. T. Shawagfeh, "Generalized Taylor's formula," Applied Mathematics and Computation, vol. 186, no. 1, pp. 286-293, 2007.

[8] K. Diethelm, The Analysis of Fractional Differential Equations, Springer, Berlin, Germany, 2010.

[9] D. Funaro, Polynomial Approximations of Differential Equations, Springer, 1992.

[10] J. S. Hesthaven, S. Gottlieb, and D. Gottlieb, Spectral Methods for Time-Dependent Problems, Cambridge University, 2009.

[11] P. Mokhtary and F. Ghoreishi, "The $L^{2}$-convergence of the Legendre spectral tau matrix formulation for nonlinear fractional integro differential equations," Numerical Algorithms, vol. 58, no. 4, pp. 475-496, 2011.

[12] I. V. Andrianov and J. Awrejcewicz, "Analysis of jump phenomena using Padé approximations," Journal of Sound and Vibration, vol. 260, no. 3, pp. 577-588, 2003.

[13] I. Andrianov and J. Awrejcewicz, "Solutions in the Fourier series form, Gibbs phenomena and Padé approximants," Journal of Sound and Vibration, vol. 245, no. 4, pp. 753-756, 2001.

[14] I. V. Andrianov and J. Awrejcewicz, "Iterative determination of homoclinic orbit parameters and Padé approximants," Journal of Sound and Vibration, vol. 240, no. 2, pp. 394-397, 2001. 
[15] G. Kudra and J. Awrejcewicz, "Tangents hyperbolic approximations of the spatial model of friction coupled with rolling resistance," International Journal of Bifurcation and Chaos, vol. 21, pp. 2905-2917, 2011.

[16] O. D. Makinde, "Solving microwave heating model in a slab using Hermite-Padé approximation technique," Applied Thermal Engineering, vol. 27, pp. 599-603, 2007. 


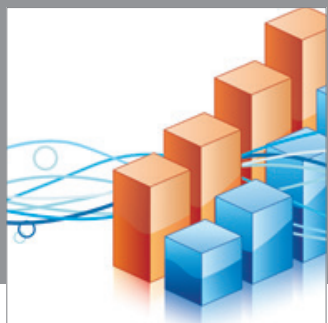

Advances in

Operations Research

mansans

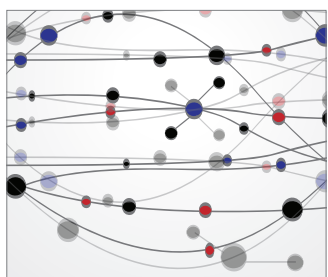

The Scientific World Journal
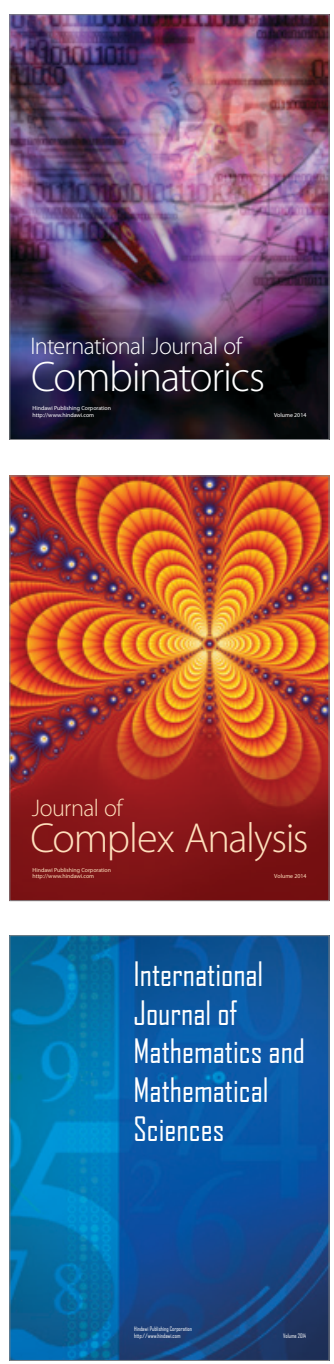
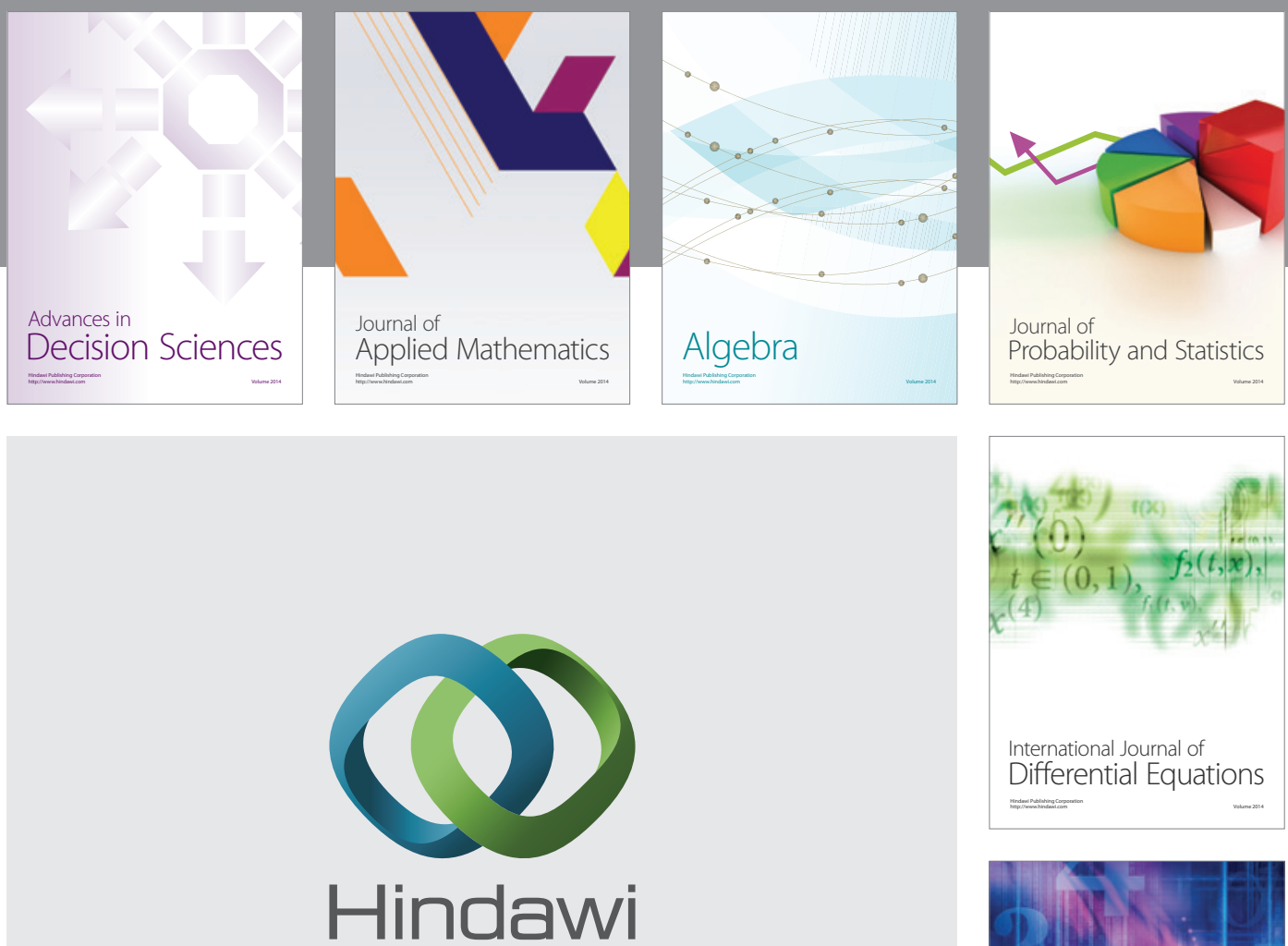

Submit your manuscripts at http://www.hindawi.com
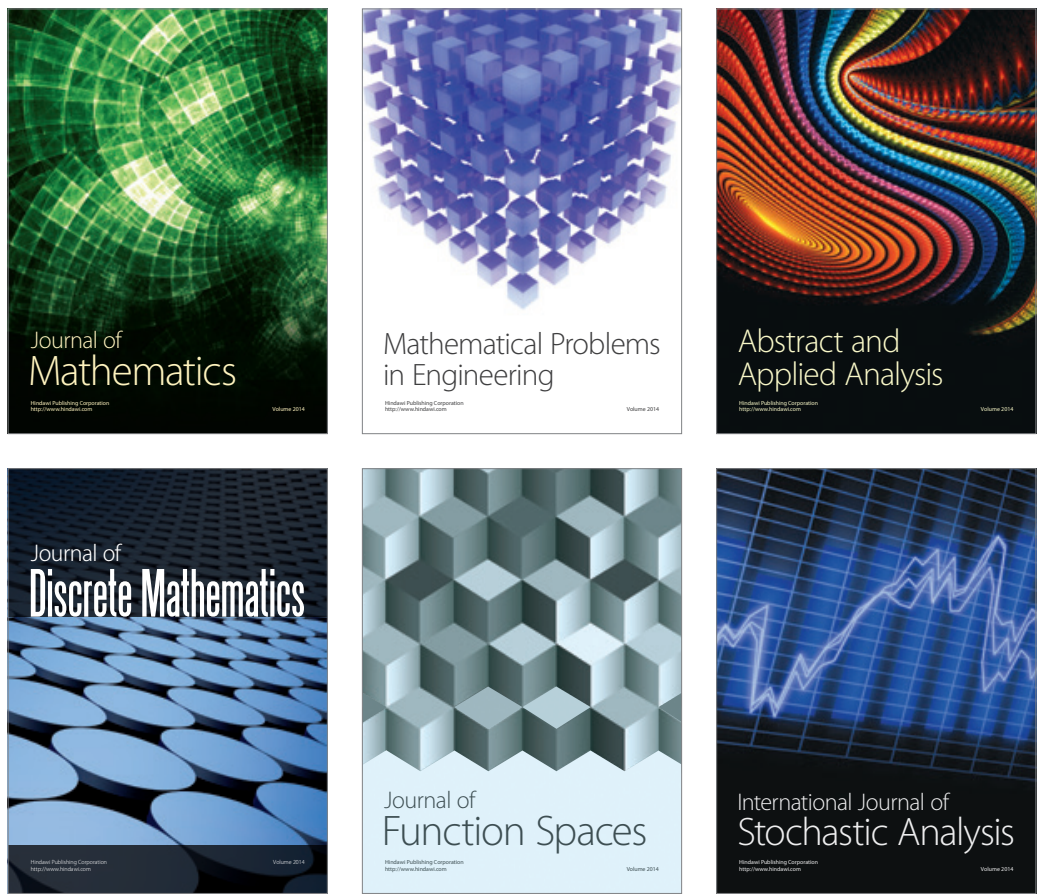

Journal of

Function Spaces

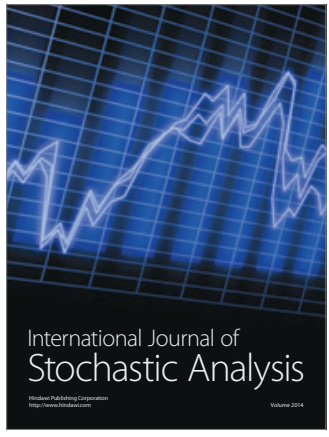

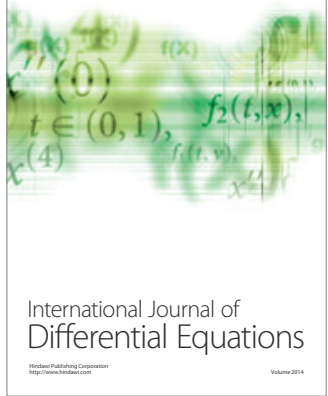
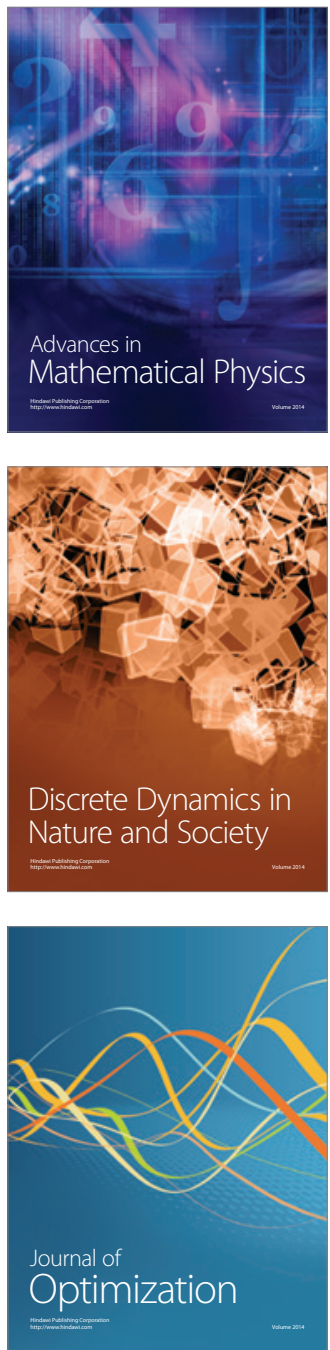